\title{
Fundamental concepts of measurement
}

\author{
L. Finkelstein \\ The City University, Department of Systems and Automation, St. John Street, London E.C.1., England
}

This is a reissue of a paper which appeared in ACTA IMEKO 1973, Proceedings of the $6^{\text {th }}$ Congress of the International Measurement Confederation, "Measurement and instrumentation", 17-23.6.1973, Dresden, vol. 1, pp. 11-27.

The paper proposes a clear, synthetic presentation of some basics of the measurement science.

\section{Section: RESEARCH PAPER}

Keywords: measurement theory, uncertainty

Citation: L. Finkelstein, Fundamental concepts of measurement, Acta IMEKO, vol. 3, no. 1, article 4, May 2014, identifier: IMEKO-ACTA-03 (2014)-01-04

Editor: Luca Mari, Università Carlo Cattaneo

Received May $1^{\text {st }}, 2014 ;$ In final form May $1^{\text {st }}, 2014$; Published May 2014

Copyright: $\odot 2014$ IMEKO. This is an open-access article distributed under the terms of the Creative Commons Attribution 3.0 License, which permits unrestricted use, distribution, and reproduction in any medium, provided the original author and source are credited

\section{THE IMPORTANCE OF MEASUREMENT SCIENCE}

The importance of measurement in natural science and technology is undeniable. Measurement is the essential tool of scientific investigation and discovery, and it enables the complex phenomena of the universe to be described in the precise, concise and universal language of mathematics, without which it would, in general, be impossible to deduce laws from scientific observation or to formulate useful theoretical constructs. In technology, the increasing complexity and speed of many modern processes and machines, make automatic control essential, and such control is not possible without satisfactory means of measurement.

The last three decades have seen spectacular advances in the technology of measurement of physical quantities. The development of electrical sensors and electronic data handling have vastly extended the range of what can be measured.

Measurement is also making spectacular advances in areas in which it had not hitherto been fruitfully or extensively applied. The social, political, economic and behavioural sciences are to an increasing extent adopting quantitative techniques. The size and complexity of modern society provides difficult problems of planning and control and such activities require data derived from measurement which modern computers make increasingly possible to acquire and handle.

Yet in spite of the general recognition of the importance of measurement, the progress in its techniques and the spread of its practical application, there is widespread neglect of its fundamental problems. We have not seen a development of Measurement Science as a widely recognised, distinct branch of knowledge. The teaching of the principles of measurement is neglected in many academic curricula and this neglect is, if anything, increasing.

The question must be asked whether a Measurement Science as an organised, systematic body of knowledge setting out to embrace all aspects and fields of measurement is needed, and, if needed, whether it is possible to build one.

It might appear superficially that the impressive development of the technology of measurement achieved by pragmatic approaches shows that a theoretical structure of Measurement Science is to a large extent superfluous. The opposite, however, is true. The very wealth of subject matter and the explosive growth of scientific information in the field mean that this material must be systematically organised and general principles derived. Without a theoretical framework measurement technology would be just a catalogue of techniques and instruments, which a student would find too vast to assimilate, while the practising scientist or engineer would find it impossible to retrieve the right information to lead him to optimal solutions of problems. Studies of measurement without a firm theoretical basis do not have the intellectual rigour to justify their place in the academic curriculum, which their practical importance demands.

Granted that there is a need for a Measurement Science, is it possible to formulate principles and methods which have general application to all measurement and which are not trivial platitudes? The answer must be, that any measurement problem requires a scientific understanding of the particular phenomenon or process under investigation, and generally the bringing together of a wide range of technologies to its solution such as fine-mechanics, electronics, nucleonics and the like. But, while each field of measurement has its own peculiar 
features, and while measurement in one or other of its applications involves virtually all other technologies, a general principle of measurement, which are valid and useful, can be formulated, and indeed, have been, developed to a high degree of sophistication.

The principles of measurement are scattered in the literature of a variety of fields: Philosophy of Science, Statistics and, above all, that body of knowledge which is becoming known as Systems Science and Engineering. It is important to collect these principles together, to synthesize them into a coherent whole, and to bring them to bear in a fruitful way on the problems of the Technology of Measurement.

\section{SCOPE AND NATURE OF PRESENT SURVEY}

Current development of Measurement Science by engineers has in the main proceeded along two major lines. One is the treatment of measurement information by the methods of information and signal theory. The other is the systemic analysis of measuring instruments and systems using the approach of dynamic systems analysis and of general network theory. The essence of these approaches is the concept that measuring systems operate by the transformation according to a prescribed functional relation of a measurand into an observable.

It would be tempting to try to survey the general structure of Measurement Science as it stands at present. Such an approach, however, would be too superficial to be useful. It is proposed therefore to examine one aspect of the subject only, its logical foundations and epistemology. The paper will attempt to review our understanding of the way in which we create an image of reality in terms of numbers and signals, a step which must precede the subsequent processing of the information.

Even the relatively limited topic tackled is too wide to be treated in this review with absolute rigour nor can all controversial philosophical points be critically discussed. All that is proposed is to outline key concepts as they are seen by the author.

For detailed discussions and comprehensive bibliographies readers are referred to references [1-7].

\section{HISTORICAL DEVELOPMENT OF THE EPISTEMOLOGY OF MEASUREMENT}

The logical foundations of measurement - the relation between the material universe and mathematics - have been studied since the dawn of science. One may perhaps date interest in the subject from Pythagoreans and their view that all things are number. Aristotle presented the first analysis of the philosophical problems of measurement in his Metaphysics. Plato made a distinction between pure arithmetic and. its application to the real world and this greatly influenced subsequent thinkers. Platonic idealism diverted attention from the problems of logical analysis of measurement. A major landmark in the progress of understanding of the relations between numbers and the real world has been the establishment by Descartes of the connection between algebra and geometry. Newton was also concerned with the logical foundations of measurement in his development of the theory of fluents.

The foundations of the modern study of the epistemology of measurement with reference mainly to Physics have been laid down by Helmholtz [8] in the last century. This work has been extended and developed in the twenties of this century in the lucid writings of Campbell $[9,10]$.
Mathematicians concerned with the logical basis of their subject have naturally been concerned with the axioms of quantification. Russell [11], Frege [12], Hoelder [13] and Wiener [14] have among others explored this topic.

Philosophers of science have also given measurement their attention in recent times within the general context of their subjects. It may be invidious to single out particular contributions but one can perhaps mention the work of Carnap [15], Cohen, Nagel [16], Suppes, Zinnes [7] and Ellis [1].

The logical foundations of measurement have been involved in the studies of the theory of dimensions by such writers as Bridgman [17], Wallot [18], Stille [19] and others.

The principal problems that have motivated the modern development of the study of the logical foundations of measurement have been above all the need to develop the methods of measurement in psychology, with which the classical approach of Helmholz could not deal, since it was based on the possibility of additive combination of quantities, which cannot be achieved in the case of say psychophysical responses to stimuli. The work of Stevens [20], Thurstone [21], Torgerson [22], Suppes and Zinnes [7] among others, have in dealing with psychological problems extended the frontiers of our understanding of measurement against, often, the conservative opposition of physical scientists.

Another area where the need for quantification is growing is that of Econometrics and Utility Theory. This has led among others to the work on measurement theory of Pfanzagl [2].

This brief historical note is not intended to be comprehensive or to evaluate critically the individual contributions of the various workers but merely to provide a sketch of the antiquity of the problem, and the wide circles of workers concerned with it, to give credit to some of the principal contributors and to mention a few key publications.

\section{EPISTEMOLOGY OF MEASUREMENT AND THE TECHNICAL SCIENCES}

In spite of the importance of measurement in technology logical analysis of measurement has received virtually no treatment in the literature of technical measurement. Discussions of the subject by engineers are rare. The view is often held that the problems of epistemology of measurement are of no practical interest in technology since the nature of technical measurement is self evident and satisfactorily understood.

It is important to assert the opposite in this survey. In automation of industrial processes one of the outstanding practical problems is the monitoring by automatic means of complex product properties, such as flavour of foods or appearance of a decorative surface. It is by no means obvious how one can express such qualities in numbers. The practical solution of the problem depends upon the proper application of the epistemology of measurement.

Again in the design of engineering systems especially in technical cybernetics it is commonly necessary to formulate performance criteria which quantify various system characteristics and combine these quantities with different weightings so as to form a measure of the total utility of the design. A sound approach to this neglected problem requires an understanding of the fundamental nature of quantification.

Finally in the problem of error analysis and especially in the Bayesian approach, the crucial question is the determination of measures which express correctly the unsatisfactoriness of 
erroneous estimates. This requires again an understanding of the philosophy of measurement.

\section{DEFINITION OF MEASUREMENT}

Measurement is the assignment of numbers to entities in such a way as to describe them.

I propose to define a number in the sense in which I am going to use the word, as an element $\mathrm{n}$ of a set of symbols $\mathrm{N}$, which has a structure or set of relations defined on it.

Consider a set of extra mathematical entities $Q$ and a set of numbers $\mathrm{N}$. Measurement is an operation which assigns to an element $\mathrm{q}_{\mathrm{i}} \in \mathrm{Q}$, an element $\mathrm{n}_{\mathrm{i}} \in \mathrm{N}$, such that relations between different elements $n_{i} \in N$ and $n_{j} \in N$ are isomorphic to empirical relations between the corresponding elements of $\mathrm{Q}, \mathrm{q}_{\mathrm{i}}$ and $\mathrm{q}_{\mathrm{j}}$.

\section{CONCEPTUAL BASIS}

Measurement presupposes something to be measured. Both in the historical development and logical structure of scientific knowledge, measurement requires a clear operational defining concept of the class of entities described by it, that is a rule for determining whether an entity is a member of the class. Unless there exists such a clear concept, we cannot start to measure.

As precise knowledge derived from measurement accumulates, this usually leads to a clearer and more satisfactory concept definition. The process is iterative and the development is an ascending spiral.

In some cases the concept of an entity arises from numerical laws, arrived at by measurement, and the entity is best thought of in such mathematical terms, but in general one attempts to arrive at some qualitative conceptual framework for it, if possible.

One of the principal problems of scientific method is to ensure that the scale of measurement established for a class of entities yields measures, which in all contexts, describe the entity in a manner which corresponds to the underlying concept of the class.

\section{DIRECT MEASUREMENT}

Direct measurement is the process of measuring an entity by comparison with entities of the same class and without reference to the measurement of any other class of entities. It relies on the establishment on the class of entities Q, upon which a scale of measurement is to be defined, of a system of empirical relations $\mathrm{R}$, which have a formal similarity to relations among members of the class of numbers $\mathrm{N}$.

It is proposed to explain the process of direct measurement by examples.

The simplest form of $\mathrm{R}$ is $\mathrm{R}=\{\mathrm{Q}, \sim\}$ in which we establish on $\mathrm{Q}$ an equivalence relation $\sim$. An equivalence relation is one which is reflexive $(\mathrm{q} \sim \mathrm{q})$, symmetrical (if $\mathrm{q}_{1} \sim \mathrm{q}_{2}$ then $\mathrm{q}_{2} \sim \mathrm{q}_{1}$ ) and transitive (if $\mathrm{q}_{1} \sim \mathrm{q}_{2}$ and $\mathrm{q}_{2} \sim \mathrm{q}_{3}$ then $\mathrm{q}_{1} \sim \mathrm{q}_{3}$ ). An equivalence relation has the formal properties of equality.

Given $R=\{Q, \sim\}$ a set of differing entities $s_{i} \in Q$ may be selected to form a standard set $\mathrm{S}=\left\{\mathrm{s}_{1}, \mathrm{~s}_{2}, \ldots \mathrm{s}_{\mathrm{k}}\right\}$.

Numerals or other symbols $\mathrm{n}_{\mathrm{i}} \in \mathrm{N}$ are assigned to each $\mathrm{S}_{\mathrm{i}}$, the same symbol not being assigned to two different standards. In measurement entities $\mathrm{q} \in \mathrm{Q}$ are compared with elements of $\mathrm{S}$ and those which bear the relation $\sim$ to a standard are assigned the same symbol as the standard. This assignment constitutes nominal measurement. An example of such measurement is the use of a colour chart based on the empirical relation of "colour matching".

Class inclusion is an equivalence relation. Consider that $Q$ can be divided by empirical operations into n classes $\mathrm{Q}_{\mathrm{i}} \subset \mathrm{Q}$ such that $\cup_{i=1}^{n} Q_{i}=Q$ and $Q_{i} \cap Q_{j}=\varnothing$ if $i \neq j$ and each subset $Q_{i}$ is assigned a unique number say "i". Then if we can determine by an empirical operation that any element $q \in Q$ belongs to a particular subset $\mathrm{q} \in \mathrm{Q}_{\mathrm{i}}$, it is assigned the characteristic symbol or number of the subset "i". The subset $\mathrm{Q}_{\mathrm{i}}$ may itself often be divided into further subsets, $\mathrm{Q}_{\mathrm{i}, \mathrm{j}} \subset \mathrm{Q}_{\mathrm{i}}$, $\cup_{j=1}^{n} Q_{i, j}=Q_{i}$ and $Q_{i, j} \cap Q_{i, k}=\varnothing$ if $j \neq k$. The subsets $Q_{i, j}$ are assigned unique numbers, say “ij”. An element for which we assign the class inclusion relation $q \in Q_{i, j}$, is assigned the symbol "ij" which denotes its membership of $Q_{i}$ and $Q_{i, j}$. This can be continued to an even finer subdivision. Classificatory schemes of this kind such as decimal library classifications constitute a form of nominal measurement.

It is often disputed whether the processes just described can in fact be called measurement. Measures on a nominal scale merely describe whether two entities are identical or different. It is immaterial whether we term nominal measurement proper measurement or not, provided we recognise its similarity, to more sophisticated forms of quantification. It is very different from naming, which is labelling without description. Two individuals may have the same name without being in any way similar, but two entities with the sane nominal measure are related.

More elaborate than the nominal is the ordinal scale based on the establishment on $\mathrm{Q}$ of an empirical order system $\mathrm{R}=\{\mathrm{Q}, \sim, \prec, \succ\} . \sim$ is an equivalence relation. The set of relations is complementary, that is for any $\mathrm{q}_{1}, \mathrm{q}_{2} \in \mathrm{Q}$, one and only one of the following must hold: $\mathrm{q}_{1} \sim \mathrm{q}_{2}, \mathrm{q}_{1} \prec \mathrm{q}_{2}$ or $\mathrm{q}_{1} \succ \mathrm{q}_{2}$. $\prec$ and $\succ$ are converse (that is if $\mathrm{q}_{1} \prec \mathrm{q}_{2}$ then $\mathrm{q}_{2} \succ \mathrm{q}_{1}$ ), irreflexive (that is we cannot have $\mathrm{q} \prec \mathrm{q}$ ) transitive and asymmetrical (that is if $\mathrm{q}_{1} \prec \mathrm{q}_{2}$ then not $\mathrm{q}_{2} \prec \mathrm{q}_{1}$ ). The relation system $\mathrm{R}=\{\mathrm{Q}, \sim, \prec, \succ\}$ enables entities of class $\mathrm{Q}$ to be arranged in an ordered series. Entities that can be so arranged may be called quantities.

We can now select a number of differing standard entities $\mathrm{s}_{\mathrm{i}} \in \mathrm{Q}$ and arrange them in an ordered standard series $\mathrm{S}=\left\{\mathrm{s}_{1}, \mathrm{~s}_{2}, \ldots \mathrm{s}_{\mathrm{k}}\right\}$. Numerals, are assigned to each $\mathrm{s}_{\mathrm{i}}$, say $\mathrm{i}$, in such a way that the order of numerals corresponds to the order in the standard series of the standards to which they are assigned. Any entity $\mathrm{q} \in \mathrm{Q}$ can then be measured by comparing it with the elements of $S$ in the same way as in nominal measurement. If $\mathrm{q}$ bears the relation $\sim$ to any element $\mathrm{s}_{\mathrm{i}} \in \mathrm{S}$ it is assigned the numeral of $\mathrm{s}_{\mathrm{i}}$. If an entity is not equivalent to any of the standards it is possible to determine between which two standards the measured entity would lie in the standard series. It is then assigned a numeral lying in between those of the two standard entities. The best example of an ordinal scale of measurement is the Mohs' scale of hardness of minerals. 
Neither nominal nor ordinal measurement as described here constitute entirely satisfactory measurements. In general measurement scales are designed to define a distance concept on the scale of entities to be measured.

The analysis and axiomatisation of the problem of defining such a distance concept has received much attention in the literature. Those interested are referred to the works listed in the bibliography. It is proposed here to outline the classical approach originating from the work of Helmholtz.

Basically this depends upon defining on the class $\mathrm{Q}$, in addition to an order relation system $\mathrm{R}=\{\mathrm{Q}, \sim, \prec, \succ\}$, a binary operation $\mathrm{C}$ which combines two entities of the class to form a third entity of the class:

$$
\begin{aligned}
& \mathrm{C}\left(\mathrm{q}_{1}, \mathrm{q}_{2}\right) \sim \mathrm{q}_{3} \\
& \mathrm{q}_{3} \succ \mathrm{q}_{1} \quad \mathrm{q}_{3} \succ \mathrm{q}_{2}
\end{aligned}
$$

The combination must be commutative:

and associative:

$$
\mathrm{C}\left(\mathrm{q}_{1}, \mathrm{q}_{2}\right) \sim \mathrm{C}\left(\mathrm{q}_{2}, \mathrm{q}_{1}\right)
$$

$$
C\left(\mathrm{q}_{1}, \mathrm{C}\left(\mathrm{q}_{2}, \mathrm{q}_{3}\right)\right) \sim \mathrm{C}\left(\mathrm{C}\left(\mathrm{q}_{1}, \mathrm{q}_{2}\right), \mathrm{q}_{3}\right)
$$

These are the formal properties of addition for the class of real numbers.

For two masses, for example, rigid connection is a form of combination which meets the requirements specified above, and the equiarm balance is the means by which the relations 'greater than', 'less than' and 'equal to' are established.

When such a method of combination has been found, a single entity $\mathrm{s}_{1} \in \mathrm{Q}$ is chosen as standard and assigned the number 1. Another entity $s_{1}^{1} \in Q$ such that $s_{1}^{1} \sim s_{1}$ is then sought and $C\left(s_{1}^{1}, s_{1}\right) \sim s_{2}$ is assigned the number 2 .

We then proceed to form $C\left(\mathrm{~s}_{2}, \mathrm{~s}_{1}\right) \sim \mathrm{s}_{3}$ and assign it the number 3 and so on. Fractional standards are generated by making or seeking two entities $\mathrm{s}_{1 / 2}, \mathrm{~s}_{1 / 2}^{1} \in \mathrm{Q}$ such that $\mathrm{s}_{1 / 2} \sim \mathrm{s}_{1 / 2}^{1}$ and $\mathrm{C}\left(\mathrm{s}_{1 / 2}, \mathrm{~s}_{1 / 2}^{1}\right) \sim \mathrm{s}_{1}$ and assigning to $\mathrm{s}_{1 / 2}$, the number $1 / 2$. Thus we generate an extended set of standards:

$$
\mathrm{S}=\left\{\ldots \mathrm{s}_{1 / 2}, \mathrm{~s}_{1}, \mathrm{~s}_{2}, \ldots\right\}
$$

Any quantity $q \in Q$ can then be measured by seeking the element $s_{i}$ to which it bears the relation $\sim$. $\mathrm{q}$ is assigned the number corresponding to $\mathrm{s}_{\mathrm{i}}$.

Thus we can see that using the above procedure we have established a process of assigning numbers to entities in such a way that the numbers tell something of the extent by which one entity differs from another.

The above argument has eschewed rigour for the sake of simplicity, but the procedures described can be rigorously axiomatised.

Scales based on additive combination represent the classical view of fundamental measurement. They give an accurate and penetrating insight into measurement in Physics but in many areas of science they cannot be applied. The principal advances of modern theory of measurement have been to show that other methods of arriving at direct scales establishing a distance concept on a class of extra-mathematical entities with the same properties as those described above, are equally valid. One way, for instance, of establishing a scale is by the process of assigning to any pair of psychophysical stimuli a third stimulus judged to be equidistant from them.

The essence of all direct measurement scales is an establishment of an isomorphism between empirical relations on the class of measurands and the relations on a class of numbers are not confined to particular isomorphisms.

\section{INDIRECT MEASUREMENT}

While a wide variety of conceptual entities are capable of direct measurement, there are others which are not. These must be measured by scales which rely upon relations which the entities to be measured bear to other measurable quantities. This is termed indirect measurement.

Two forms of indirect measurement can be distinguished. The first of these is derived measurement. Consider a case in which all systems $\mathrm{S}$ associated with a member of the class of entities $\mathrm{Q}$, for which we are to define a scale, are also associated with quantities $x, y, z$... which can be measured. Let there be a numerical law $P(\mathrm{x}, \mathrm{y}, \mathrm{z})=\mathrm{k}_{\mathrm{q}}$ in which $\mathrm{k}_{\mathrm{q}}$ is a constant. Let us further suppose that whenever the systems $\mathrm{S}$ are arranged in the order of $\mathrm{Q}$, they are also arranged in the order of $\mathrm{k}_{\mathrm{q}}$. Then $\mathrm{k}_{\mathrm{q}}$ can be taken to be a derived measure of $\mathrm{Q}$.

Density is an example of a physical quantity measured by a derived scale. For all objects of one material the ratio $\varrho$ of mass to volume of the object is a constant. Whenever objects are arranged in order of density as qualitatively defined, they are also arranged in order of $\varrho$, which is thus a measure of density.

The other form of indirect measurement is associative. Consider that all systems $\mathrm{S}$, associated with a member of class $\mathrm{Q}$, are also associated with some other measurable quantity $\mathrm{x}$. Further suppose that whenever the systems $\mathrm{S}$ are ordered according to $\mathrm{Q}$ they are also arranged in the order of $\mathrm{x}$. We can then define $f(x)$ as an associative measure of $Q$, where $f()$ is an arbitrary, single-valued, monotonic function.

All practical temperature scales are of the associative kind.

\section{SCALES OF MEASUREMENT}

It should be clear from what has been said that it is not adequate in measurement merely to speak of units, one should speak of scales of measurement, that is of the triplet Q, M, N where $\mathrm{Q}$ is a class of entities, $\mathrm{N}$ is a class of numbers and $\mathrm{M}$ is a procedure of relating members of 0 . to members of $\mathrm{N}$.

\section{SCALE CLASSIFICATION}

Forms of scale can be classified according to mathematical transformations which leave the scale invariant.

Thus if we transform the number $\mathrm{n}$ on the scale to a number n' we have:

\section{Permissible Transformation \\ $n^{\prime}=f(n) \quad$ where $f$ any one to one Nominal \\ $\mathrm{n}^{\prime}=\mathrm{f}(\mathrm{n}) \quad \mathrm{f}$ any monotonic function $\quad$ Ordinal \\ $\mathrm{n}^{\prime}=\mathrm{an}+\mathrm{b} \quad \mathrm{a} \neq 0 \quad$ Interval \\ $\mathrm{n}^{\prime}=$ an $\quad \mathrm{a} \neq 0 \quad$ Ratio}

A permissible transformation is one which only changes that which has been arbitrarily chosen. Thus the scale of mass is a ratio scale because the only thing arbitrarily chosen is the unit mass. Similarly associative scales are generally ordinal.

\section{MEASURE AND CONCEPT}

The point has already been made that the measure of an entity must constitute a description which corresponds to the defining concept of the entity. Thus there must be isomorphism between any empirical relations among quantities 
measured and corresponding relations between their measures. One basic test of this correspondence can be proposed. If, whenever systems associated with an entity of class Q are placed in an order according to our quantitative concept of Q, the order is the same as that in which the systems would be arranged by the order of some measure of $\mathrm{Q}$, the measure can be considered satisfactory.

\section{MEANINGFULNESS}

The problem of the meaningfulness of statements made about measures of an entity is important.

A statement is meaningful if its truth is unchanged by a permissible transformation of the scales of measurement. We say that a k-ry relation $\mathrm{S}$ is meaningful if:

$$
\mathrm{S}\left(\mathrm{m}\left(\mathrm{q}_{1}\right) \ldots \mathrm{m}\left(\mathrm{q}_{\mathrm{k}}\right)\right)=\mathrm{S}\left(\mathrm{m}^{\prime}\left(\mathrm{q}_{1}\right) \ldots \mathrm{m}^{\prime}\left(\mathrm{q}_{\mathrm{k}}\right)\right)
$$

where $\mathrm{m} \rightarrow \mathrm{m}$ ' is a permissible transformation.

Thus, as a very simple example, it is meaningful to speak of the ratio of two masses, since that ratio is invariant with respect to changes of the unit of mass, but it is not meaningful to speak of the ratio of two hardnesses measured on the Mohs' scale, since that ratio would be changed by a monotonic transformation of the scale.

Another view is that only such statements are meaningful which can be logically traced to the empirical operations on which the measurement is founded.

\section{SYSTEMS OF SCALES IN PHYSICS DIMENSIONS}

There is much that is arbitrary in the choice of scale forms. The guiding principle is that scales are so chosen as to result in the greatest simplicity of the resulting mathematical descriptions of the laws of nature. Hence, for instance, the attempt to make practical temperature scale coincide with the thermodynamic temperature scale.

In Physics we attempt to reduce to the minimum the number of quantities termed primary the scales of which are independently defined.

Scales for the measurement of other quantities are termed secondary and they are obtained as derived scales from primary quantities by a chain of simple proportionality laws [1, 19].

Two points need to be made. There is nothing intrinsically fundamental in the primary quantities: mass, length, time, current and luminous intensity, they are merely conventionally chosen for reason of the practical convenience of the definition of their scales. Secondly, the dimension of a quantity in no way embodies any metaphysical essence. It merely denotes the way in which the conventionally chosen scale of the quantity relates to the conventionally chosen scale of the primary quantities and could conceivably be altered by different choice of scales.

\section{MULTIDIMENSIONAL QUANTITIES}

Certain entities cannot be described by a single measure, but require an array of numbers for their specification:

$$
\mathrm{M}(\mathrm{q})=\underline{\mathrm{n}}=\left[\mathrm{n}_{1}, \mathrm{n}_{2}, \ldots \mathrm{n}_{\mathrm{k}}\right]
$$

Such measures are known as vector or multi-dimensional. Thus force is described by three numbers: a magnitude and two direction angles, or three components. Patterns may be described by an array of numbers specifying features.

$\underline{\mathrm{n}}$ may be viewed as specifying a point in a vector space and we may seek to define a metric for a vector space, that is a relation $\mathrm{D}$, such that for any three vectors $\underline{\mathrm{n}}_{1}, \underline{\mathrm{n}}_{2}, \underline{\mathrm{n}}_{3}$ we have:

$$
\begin{aligned}
& \mathrm{D}\left(\underline{\mathrm{n}}_{1}, \underline{\mathrm{n}}_{2}\right)=0 \\
& \mathrm{D}\left(\underline{\mathrm{n}}_{1}, \underline{\mathrm{n}}_{2}\right)=\mathrm{D}\left(\underline{\mathrm{n}}_{2}, \underline{\mathrm{n}}_{1}\right) \text { if } \underline{\mathrm{n}}_{1} \neq \underline{\mathrm{n}}_{2}
\end{aligned}
$$

$$
\mathrm{D}\left(\underline{\mathrm{n}}_{1}, \underline{\mathrm{n}}_{2}\right)+\mathrm{D}\left(\underline{\mathrm{n}}_{2}, \underline{\mathrm{n}}_{3}\right)>\mathrm{D}\left(\underline{\mathrm{n}}_{1}, \underline{\mathrm{n}}_{3}\right)
$$

$\mathrm{D}(\mathrm{)}$ represents a measure of distance in the vector space.

The problem of representing some aspects of $\underline{n}$ by a single real number is important especially in decision and optimisation theory where we wish to place multidimensional utilities in some order. A typical method is to determine:

$$
\mathrm{m}^{2}=\underline{\mathrm{n}} \mathrm{A} \underline{\mathrm{n}}^{\mathrm{T}}
$$

where $A$ is a positive definite weighting matrix. It is important to establish logically what correspondence exists between $\mathrm{m}$ and the entity $\mathrm{q}$ measured by $\underline{\mathrm{n}}$.

The problems of multidimensional measures offer many still unresolved problems.

\section{MEASUREMENT AND UNCERTAINTY}

The essential assumption underlying the logic of measurement is that the operation $\mathrm{M}$ forms a single image of $\mathrm{q}$ only, and that $\mathrm{M}$ is invariant. However, any practical operation defining a scale represents a probabilistic and not a deterministic transformation. $\mathrm{M}$ is associated with a probability distribution, which must be specified with it.

This subject has not been adequately discussed in the literature.

\section{PURPOSE AND USES OF MEASUREMENT}

While measurement is generally recognised to be the foundation of science, in the context of a review of its fundamental concepts there is a need to analyse the purpose and uses of measurement critically.

The advantages of measurement as a form of description can be summarised as follows.

Firstly, measurement represents a description of an entity which is concise, telling us in a single number information which would otherwise need many words. Measurement is also a description which is precise, pinpointing by a single number a particular entity where the same verbal description indicates a range of similar but differing things.

Measurement is objective rather than subjective, that is, it constitutes a description invariant with respect to the observer making it.

The language of measurement is universal and commonly understood, though this demands much effort in the establishment and maintenance of good and generally accepted scales and standards.

Quantitative description involves an ability to make distinctions and to describe relations among entities of the same kind. In particular the ability of ranking objects in an order according to some measure of a relevant attribute, say, size, coat, or efficiency, enables us to make decisions about them in a logical and systematic manner.

A measure of an entity gives us an ability to express facts and conventions about it in the formal language of mathematics. Without the convenient notation of this language, the complex chains of induction and deduction by which we describe and explain the universe, would be too cumbersome to express. We could not make our thoughts clear either to ourselves or to others. It follows from what has been said that the description of entities by numbers is not good in itself. The only value of measurement lies in the use to which the information is put. Science is not just the amassing of numerical data, it depends upon the way in which the data are analysed and organised.

Finally in relation to technical cybernetics the essential feature of measurement is that it enables the measurand to be 
expressed in signals which can be handled by machines. Qualitative information is not machine intelligible.

\section{CONCLUSIONS}

The basic conclusions of the present review can be simply stated.

The basic concepts of measurement form an essential foundation stone for the erection of a sound Measurement Science. They now have a sound logical basis which is compatible with the methods of information science. Their understanding is essential for the solution of a range of important practical engineering problems.

\section{REFERENCES}

[1] B. Ellis, Basic concepts of measurement, Cambridge University Press, 1966.

[2] J. Pfanzagl, Theory of measurement, Physica-Verlag WurzburgVienna, 1968.

[3] C.W. Churchman, P. Ratoosh, Measurement, definitions and theories, Wiley, New York, 1959.

[4] Akad. Nauk., Ukrain S.S.R. Inst. fil. gnosseogicheskiye aspekty, Izmereniyi Naukova Dumka, Kiev, 1968.

[5] Akad. Nauk., Ukrain S.S.R. Inst. fil. metodologicheskiye problemy teorii, Izmereniyi Naukova Dumka, Kiev 1966.

[6] O.A. Melnikov, O roli uzmereniyi v processe, Poznaniya Nauka, Novosibirsk, 1968.

[7] P. Suppes, J.L. Zinnes, Basic measurement theory, in Handbook of Mathematical Psychology, John Wiley, 1963.

[8] L.V. Helmholtz, Zaehlen und messen, erkenntnisstheoretisch betrachtet, in Philosophische Aufsaetze Eduard Zeller gewidmet, Leipzig, 1887.
[9] N.R. Campbell, Physics: the elements, Cambridge, 1920.

[10] N.R. Campbell, An account of the principles of measurement and calculation, Longmans and Green, London, 1928.

[11] B. Russel, The principles of mathematics - 2nd Edition, New York, 1937.

[12] G. Frege, Grundlagen der Arithmetik, 1884.

[13] O. Hoelder, Die axiome der quantitaet und die lehre von mass, Berichte uber die Verhandlungen der koeniglich Saechsischen Gesselschaft der Wissenschaften zu Leipzig (Math.-Phys. Kiasse) 53, 1-64.

[14] N. Wiener, A new theory of measurement: a study in the logic of mathematics, Proc. of Lond. Math. Soc. Ser 2. 19, pp.181205.

[15] R. Carnap, Foundations of logic and measurement, International Encyclopedia of Unified Science, Vol. 1, Chicago, University of Chicago Press.

[16] M.R. Cohen, E. Nagel, An introduction to logic and scientific method, Harcourt, New York, 1934

[17] P.W. Bridgman, Dimensional analysis, Yale University Press, New Haven, 1931.

[18] J. Wallot, Groessengleichungen, Einheiten und Dimensionen, Leipzig, 1953.

[19] U. Stille, Messen und Rechnen in der Physik, Vieweg, Braunschweig, 1961.

[20] S.S. Steven, Mathematics, measurement and psychophysics, in S.S. Steven (ed) Handbook of experimental psychology, Wiley, New York, 1951.

[21] L.L. Thurstone, The indifference function, J. Soc. Psychol 2, 1931, pp 139-167.

[22] H.S. Torgerson, Theory and methods of scaling, Wiley, New York, 1958. 